\title{
Experimental Investigation of the Delay Time in Galactic Cosmic Ray Flux in Different Epochs of Solar Magnetic Cycles: 1959-2014
}

\author{
Krzysztof Iskra $^{1} \cdot$ Marek Siluszyk $^{2}$ (D) \\ Michael Alania ${ }^{2,3}$. Witold Wozniak ${ }^{4}$
}

Received: 2 November 2018 / Accepted: 20 August 2019 / Published online: 11 September 2019

(C) The Author(s) 2019

\begin{abstract}
In the present article, we analyze long-term changes in the intensity of galactic cosmic rays (GCRs) in different polarity epochs of the solar magnetic cycles from 1959 to 2014. Our purpose is to carry out a study of the delay time (DT) between the changes of the GCR intensity and various parameters characterizing the conditions in the heliosphere. We prove the existence of varying DTs between the changes of GCR intensity and the parameters characterizing solar activity, such as sunspot number and tilt angle. Based on our investigation, we obtained different DTs in epochs with different global solar magnetic field polarities. We conclude that the observed DTs are very important parameters for the study of GCR transport in the heliosphere.
\end{abstract}

Keywords GCR flux · GCR intensity variation - Solar activity · Heliospheric magnetic field turbulence

\section{Introduction}

The 11-year variation in the intensity of galactic cosmic rays (GCRs) is generally associated with a similar variation of the solar activity (SA); there is an anticorrelation between

$凶$ M. Siluszyk

marek.siluszyk@uph.edu.pl

K. Iskra

k.iskra@law.mil.pl

M. Alania

alania@uph.edu.pl

W. Wozniak

witek.wozniak81@gmail.com

1 Polish Air Force University, Deblin, Poland

2 Siedlce University, Siedlce, Poland

3 Institute of Geophysics, Tbilisi State University, Tbilisi, Georgia

4 Polish Gas Company, Warsaw, Poland 
them (Dorman, 1974). We analyze five sub-periods with different global solar magnetic field (GSMF) polarities in the period 1959-2014. In the time profile of the GCR intensity, we can observe a plateau for the positive polarity $(A>0)$ and a peak for the negative polarity $(A<0)$ of the GSMF. This is caused by a drift occurring due to the gradient and curvature of the regular interplanetary magnetic field (IMF) (Jokipii and Thomas, 1981). Lockwood and Webber (1984) have suggested that the 11-year variation in GCRs depends on the accumulative effects of Forbush decreases. Others have tried to explain the 11-year variation as a result of a combination of drift and globally merged interaction regions with a time-dependent model (Le Roux and Potgieter, 1995), or by the diffusion barrier with other general modulation mechanisms (Ferreira and Potgieter, 2004). In several articles (Alania et al., 2001; Alania, Iskra, and Siluszyk, 2003, 2008a,b, 2010; Siluszyk et al., 2005; Siluszyk, Iskra, and Alania, 2014; Iskra, Siluszyk, and Alania, 2015; Siluszyk et al., 2018) researchers have shown that large-scale structural changes of the solar wind (SW) magnetic turbulence in different periods of SA are general mechanisms of the 11-year variations of GCR. Based on experimental investigations we have constructed mathematical models confirming the above-mentioned mechanisms of the long-term variation (Siluszyk, Wawrzynczak, and Alania, 2011; Siluszyk, Iskra, and Alania, 2015).

In the present article, we study the characteristics of the delay times (DTs) between the intensity of GCRs, various parameters characterizing SA, and the conditions in interplanetary space. These anomalous phenomena in long-term variations have been interpreted as the result of the polarity reversal of the polar magnetic field of the Sun appearing during periods of SA maxima. It is assumed that GCRs can easily enter the heliomagnetosphere if the magnetic field of the Sun is parallel to the galactic magnetic field. Otherwise, it would be difficult for GCR particles to access the heliomagnetosphere. That is the reason why a 22-year modulation of GCRs is observed (Nagashima and Morishita, 1980a). These authors found wide hysteresis loops in odd cycles (17 and 19) and narrow loops in even cycles (18 and 20) from yearly mean intensity and sunspot number (SSN).

In addition, several authors have characterized the DTs between the intensity of GCRs and SSN. DTs in odd cycles, Cycles 17 and 19, are nine and 12 months, respectively; while DTs in even cycles, Cycles 18 and 20, are one and two months, respectively, which confirms the 22-year GCR variation.

Nagashima and Morishita (1980b) have defined DTs as the time required for the SW to carry the information on the polarity reversal to the modulation boundary to allow GCR particles to react, and as the time needed for GCRs to reach Earth by the diffusion convection process after recognizing the reversal at the boundary. This means that DTs depend on the diffusion coefficient and the distance to the modulating boundary.

Van Allen (2000) also confirmed the 22-year variation through an analysis of modulating loops in Cycles 19, 21, and 22. These authors showed that the loop area in odd cycles is several times larger than that in even ones. In addition, these authors introduced a new parameter $d C / d S$ characterizing the rate of change in the intensity of GCRs with SSN. The magnitude of $d C / d S$ is larger during the initial phases of even cycles and smaller during the beginning of odd cycles. This can explain the different DTs in these cycles according to the theory of particle drift, which was modified by Jokipii and Kota based on Ulysses data (Jokipii and Kota, 1996). Observations by Ulysses showed a strong magnetic turbulence over the solar poles that inhibits the rapid access of GCRs to the interior of the heliomagnetosphere. Cliver and Ling (2001) analyzed correlations between GCR intensity and SSN in Cycles 19, 21, and 23, and they obtained DTs of about 13 months. Also, the DT between GCR intensity and tilt angle (TA) was found to be about 15 months. The evolution of the heliospheric current sheet (HCS) during each of the successive Cycles 21, 22, and 23 is also 
noteworthy. The increase of the TA to the maximum value in each of these three cycles is remarkably similar, while the decay of the TA to low values is more gradual in odd cycles. Inceoglu et al. (2014) used the hysteresis effect to model the relationship between neutron counting rates (NCRs), an indicator of the GCR intensity, and SSN over the period that covers the four Solar Cycles 20, 21, 22, and 23. On the basis of a cross-correlation coefficient between monthly NCRs and SSN, time lags of about 5-6 months were established and calculated for NCRs in the period from 1957 to 2012. Singh, Saxena, and Tiwari (2018) analyzed the Solar Cycles: 22 and 23 (1986-2008). In their article, the SA is characterized by the coronal index (CI) and the solar flare index (SFI). These authors used the fast Fourier transform and wavelet techniques. Sierra-Porta (2018) studied Solar Cycles 21, 22, and 23 (1976-2008). GCR modulation is controlled by SA, which depends on the conditions of the heliosphere. In the latter article, SA is represented by three parameters: SSN, the flare index (FI), and the Ap index. The author used a cross-correlation analysis to find the maximum negative correlation needed to find DTs. Chowdhury, Kudela, and Dwivedi (2013) and Chowdhury and Kudela (2018) stated that there is no DT between the GCR intensity and the IMF magnitude, nor between the GCR intensity and the Ap index. The above considerations show that DTs are a complex subject that depends on many parameters. DTs are influenced, not only by particle drifts, but also by the structure of the magnetic turbulence at various periods of SA characterized by many parameters, which determine the character of diffusion and of coronal mass ejection (CME) activity.

\section{Experimental Results}

Unlike the case of previous works dealing with the DT problem, the purpose of this article is to carry out a study of the DTs between changes of GCR intensity and various parameters characterizing the conditions in the heliosphere such as SSN, TA, and IMF intensity, $B$. From 1959-2014 we took monthly data of the GCR flux from the neutron monitor (NM) at Kiel and SSN, TA, and IMF magnitude. This time period is divided into five 11-year periods, each spanning a period between the SA maximum and minimum with different polarities (positive or negative) of the solar magnetic cycle (SMC).

These five 11-year periods are further divided into ten sub-periods for analysis in five different cases. In Figure 1, we present time profiles of the inverted intensity of the GCR flux and from the NM at Kiel in the 1959-2014, divided into five sub-periods corresponding to different polarities of the SMC, and the SSN.

The correlation coefficient, $r$, between the GCR intensity, I(GCR), and each of the SA parameters, SSN, TA, and $B$, was calculated to examine the DTs. This was done for each of the five 11-year time periods, each one having a different GSMF polarity. In Figures 2-6, time profiles of monthly data of the SSN and inverted intensity of GCR from the Kiel NM in five periods are presented.

\section{Methodology}

The correlation coefficient, $r$, between monthly data of the GCR flux and each of the SA parameters, SSN, TA, and B, was calculated for the considered periods, with various monthly shifts: $\Delta t=0,1,2, \ldots, 18$ months, to study the DT.

For instance, we calculated the correlation coefficient, for two independent series of input data of SSN and GCR. Next, I(GCR) data was shifted in $0,1,2, \ldots, 18$ monthly steps 


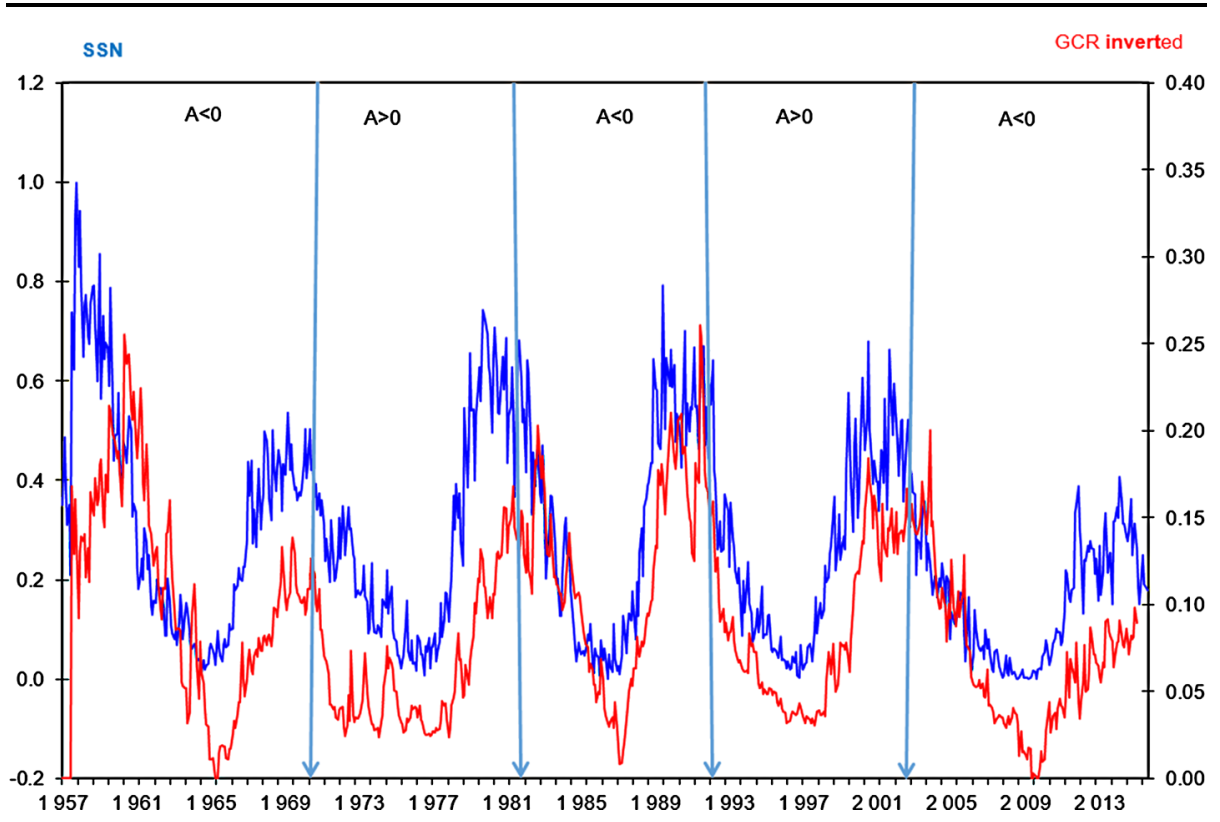

Figure 1 Time profiles of the monthly inverted intensity of GCRs from the Kiel NM and SSN. Blue arrows indicate each subsequent 11-year time period with different global magnetic solar field (GSMF) polarity. The data cover from 1959 to 2014.

relative to the SSN input data and again we calculated $r$ after every step. In each of the five periods, the DT was determined at each step when the correlation coefficient, $r$, reached the maximum value,

$$
\left[r\left(\mathrm{SSN}, I_{i}(\mathrm{GCR})\right) \rightarrow \max \right] \mathrm{DT},
$$

where $i=0,1,2,3, \ldots, 18$ is the number of shifted months.

In Table 1, and Figures $7 \mathrm{a}$, and $7 \mathrm{~b}$, we present sample correlation coefficients $r\left(\mathrm{SSN}, I_{i}(\mathrm{GCR})\right)$ for the periods $1959-1969(A<0)$ and $1991-1999(A>0)$.

We used the same method for the parameters $I(\mathrm{GCR})$, SSN, TA, and B (see Table 2). Table 2 presents the correlation coefficients and DTs in five different periods.

In Table 2 and Figures 2-6, one can see that the DTs between SSN and I(GCR) and between SSN and $B$ are larger in $A<0$ periods than in $A>0$ periods.

At the same time, we do not observe significant DTs between $B$ and $I(\mathrm{GCR})$. We also see significant correlations between the remaining parameters with various DTs; however, we do not observe a clear dependence of the DTs on the SMC polarity $(A>0$ and $A<0)$. For detailed examination of the DTs, each of the five periods with a given polarity of SMC has been divided into two sub-periods: ascending and descending (marked with an upward and downward arrow, respectively) regarding the GCR intensity. Table 3 shows $r$ and DT between each of the six pairs calculated for these ten sub-periods.

In Table 3, we observe a 22-year periodicity of the DT in the correlation between SNN and $I(\mathrm{GCR})$, but we do not observe such periodicity in the correlation between other parameters, despite the high correlation between them. On the contrary, from DT (SSN, I (GCR)) in Table 3, one can see that in $A<0$, SMC is larger in the GCR ascending period than in the descending one. For SMC where $A>0$, there is no significant DT observed in the ascending 


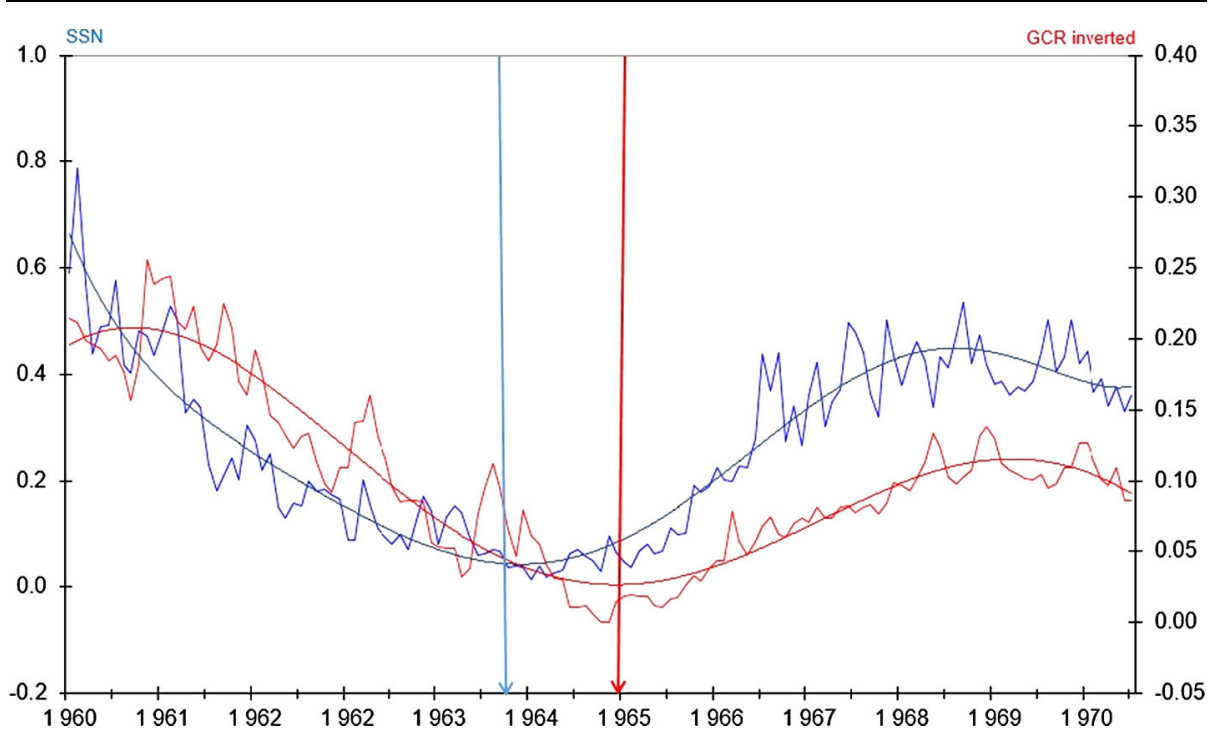

Figure 2 Time profiles of monthly data of the SSN and inverted intensity of GCR from the Kiel NM. Blue and red curves represent an approximation with a polynomial of the fifth degree of SSN data and GCR inverted data, respectively, in the period 1959-1970 for $A<0$. Blue and red arrows show a minimum value of SSN and GCR inverted data, respectively. The interval between the arrows represents a DT.

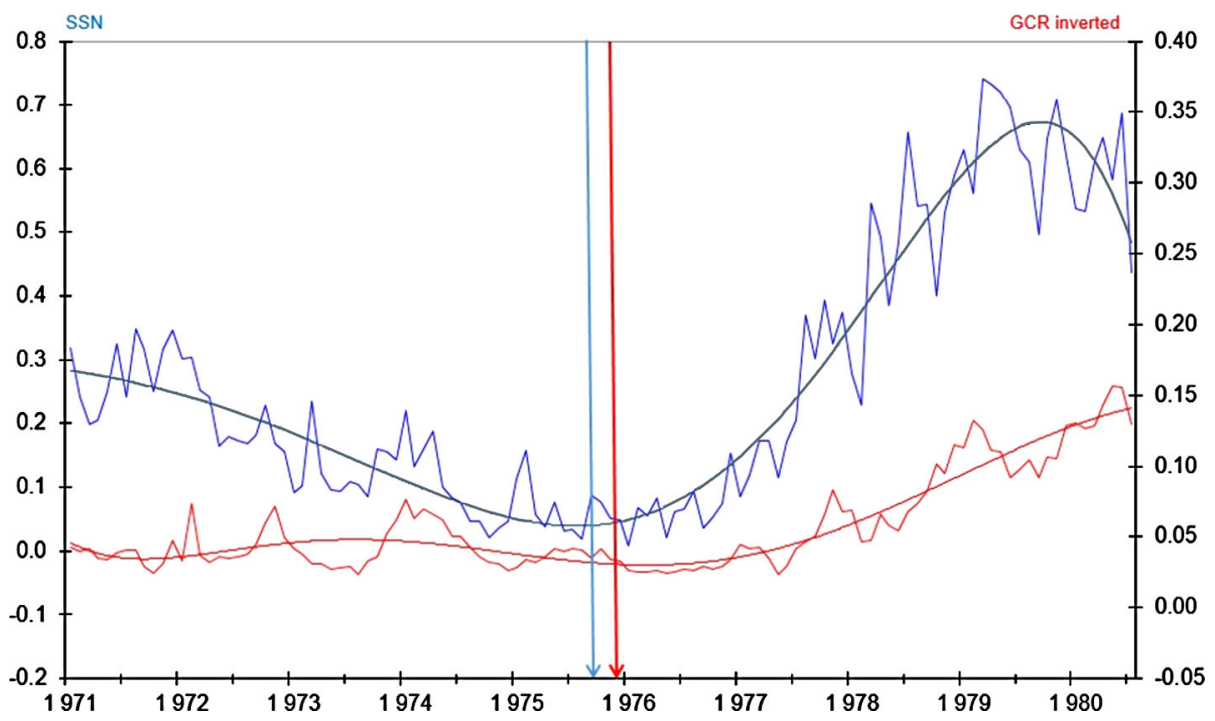

Figure 3 Similar to Figure 2 in the $1971-1979$ period for $A>0$.

period, but a small DT is observed in the descending period. In the next section, we discuss these results. 


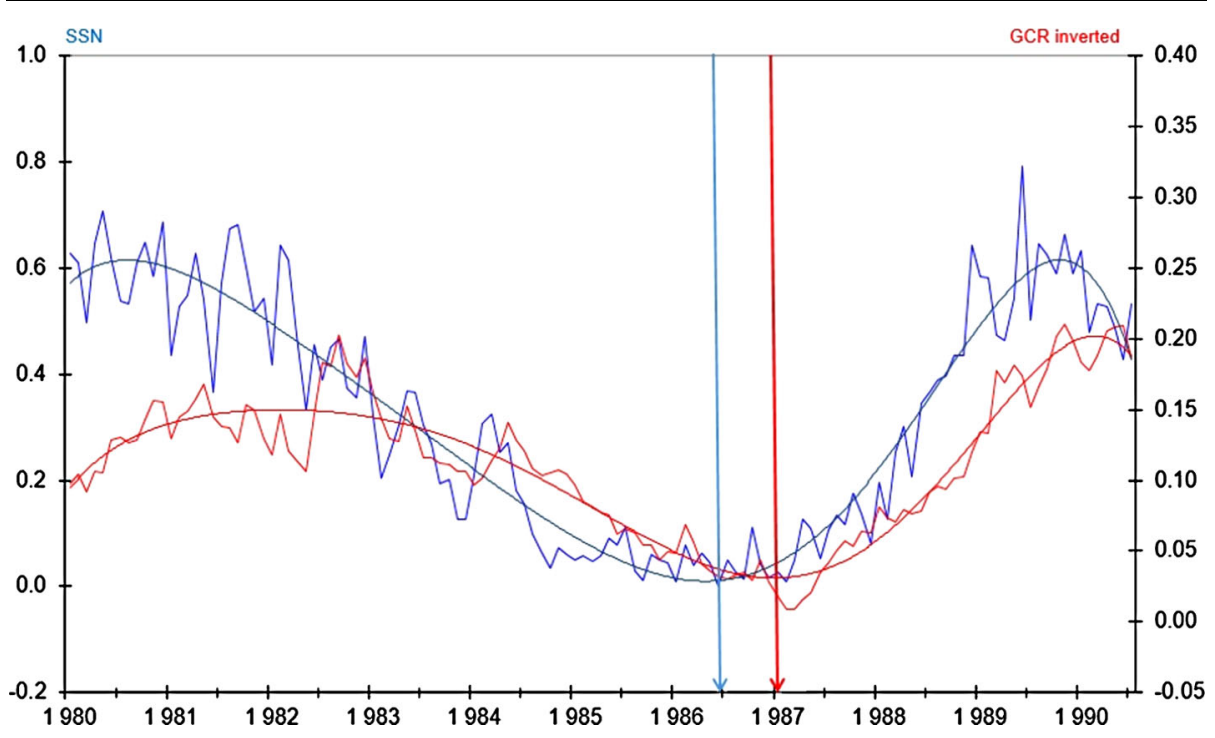

Figure 4 Similar to Figure 2 in the $1981-1990$ period for $A<0$.

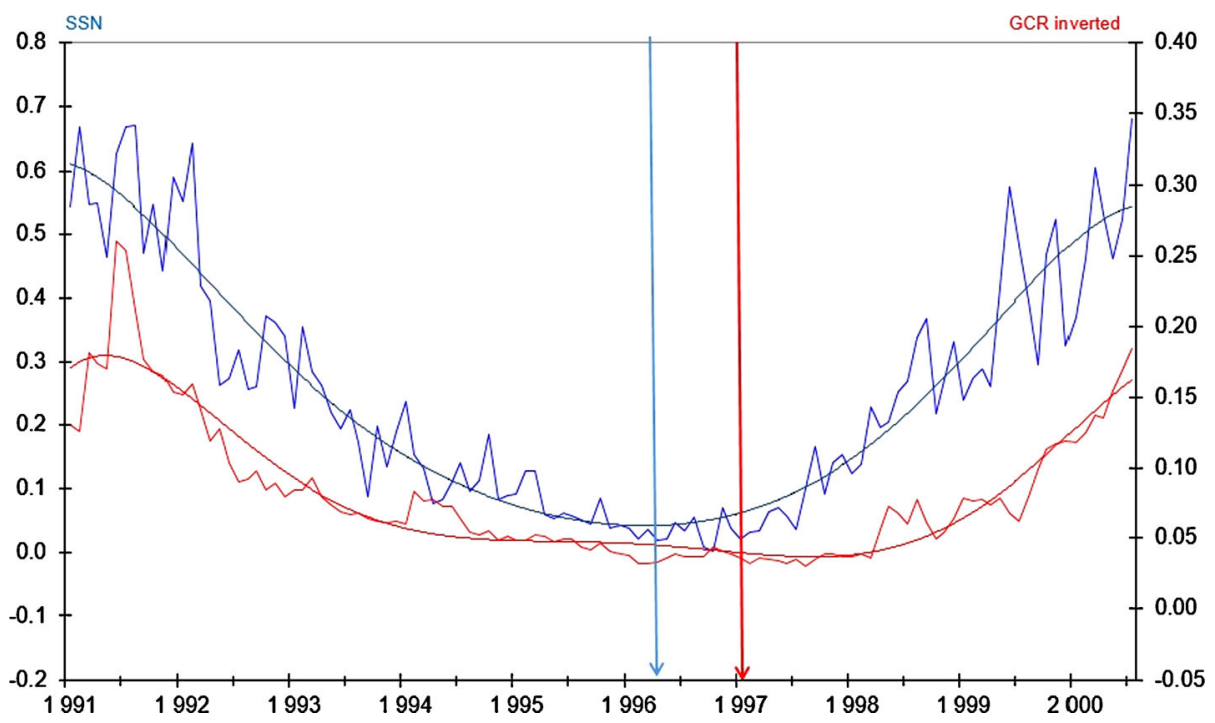

Figure 5 Similar to Figure 2 in the 1991-2002 period for $A>0$.

\section{Discussion and Interpretation of the Results}

Analyzing the correlations between changes of the GCR intensity and various parameters (see Table 3) representing the conditions in the heliosphere, we find that there is a certain regularity between SSN changes and $I(\mathrm{GCR})$; particularly, we find the 22-year variation of the DT, while we do not recognize anything similar in the correlations between other parameters. Each cycle of SA contains two periods with different polarity of the GSMF. In Cycles 20, 22, and 24, we observe a small DT (one-five months) in periods with $A<0$ and 


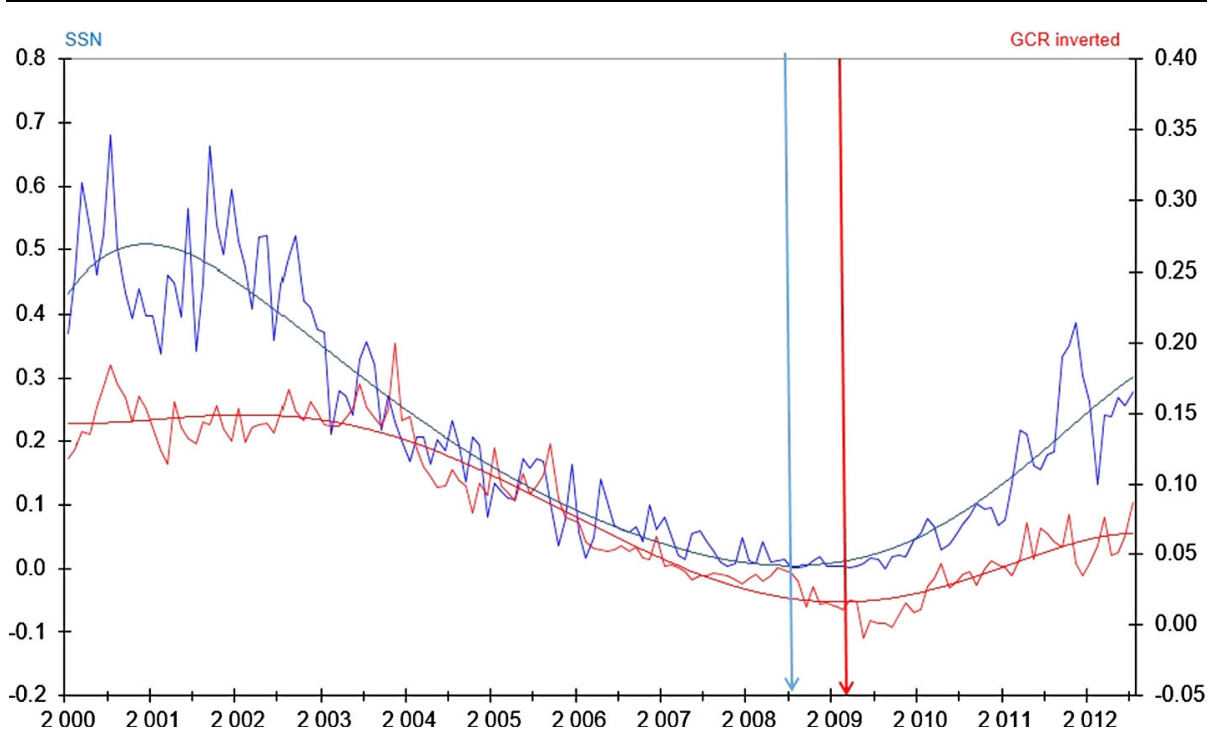

Figure 6 Similar to Figure 2 in the 2003-2013 period for $A<0$.

with descending GCR intensity, while no significant DT is observed in periods with $A>0$ and ascending GCR intensity, which consequently results in small DTs in even SA cycles. In turn, in the odd SA Cycles 19, 21, and 23, we observe a large DT in periods with $A<0$ and with ascending $I(\mathrm{GCR})$, while in periods with $A>0$ and descending $I(\mathrm{GCR})$, we observe a small DTs (one-five months), which consequently results in large DTs in odd SA cycles. The DT between $I(\mathrm{GCR})$ and each of the other parameters is very important regarding different periods of SA. The mechanism for the transport of GCRs in the heliosphere is governed by four major processes: diffusion, convection, adiabatic energy changes, and drift caused by the gradient and curvature of IMF. Spatial diffusion is caused by pitch angle scattering of GCRs by IMF turbulence. All these processes are vital, but their contribution in the modulation is different and depends on the SA. Many studies of the DT problem showed that the DTs are more pronounced in odd SA cycles (Burlaga, McDonald, and Ness, 1993; Parker, 1963) than in even SA cycles (Dorman, 1959; Jokipii and Thomas, 1981) and interpreted this phenomenon in terms of drift effects (Cliver and Ling, 2001; Chowdhury, Kudela, and Dwivedi, 2013; Chowdhury and Kudela, 2018; Mavromichalaki, Belehaki, and Rafios, 1998).

Based on the drift theory of Jokipii (1971) of the modulation of GCR in the $A>0$ epoch, a drift stream of GCRs caused by the gradient and curvature of the IMF preferentially enters the heliosphere from the polar region and is ejected outward along the equatorial current sheet.

The reverse situation occurs in the $A<0$ epoch, where the drift stream of GCRs enters the heliosphere along the HCS and exits the polar region. In the $A<0$ epoch, GCR particles measured at the Earth are more affected by propagating diffusive barriers associated with SA and the waviness of HCS, resulting in large DTs.

The opposite situation is observed in the $A>0$ epoch. GCR particles measured at the Earth are less affected by propagating diffusive barriers which causes small or insignificant DTs.

Cliver and Ling (2001) showed 22-year patterns in the relationship of SSN with TA and $I(\mathrm{GCR})$. Based on the analysis of the correlations, the authors noted that in odd cycles (19 
Table 1 Correlation coefficients, $r$, between GCR intensity, I(GCR), and SSN at every monthly shift, $i$. Shaded cells show the maximum value of $r$ for the considered period (see text).

\begin{tabular}{lll}
\hline $\begin{array}{l}\text { Number of } \\
\text { shifted months }\end{array}$ & $1959-1969$ & $1991-1999$ \\
$i$ & $r\left(\mathrm{SSN}, I_{i}(\mathrm{GCR})\right)$ & $r\left(\mathrm{SSN}, I_{i}(\mathrm{GCR})\right)$ \\
\hline 0 & -0.62 & -0.88 \\
1 & -0.65 & -0.88 \\
2 & -0.68 & -0.90 \\
3 & -0.71 & -0.92 \\
4 & -0.74 & -0.94 \\
5 & -0.76 & -0.94 \\
6 & -0.78 & -0.93 \\
7 & -0.80 & -0.91 \\
8 & -0.81 & -0.88 \\
9 & -0.82 & -0.84 \\
10 & -0.84 & -0.79 \\
11 & -0.85 & -0.76 \\
12 & -0.86 & -0.73 \\
13 & -0.87 & -0.70 \\
14 & -0.88 & -0.66 \\
15 & -0.89 & -0.63 \\
16 & -0.89 & -0.59 \\
17 & -0.89 & -0.55 \\
18 & -0.88 & -0.52 \\
19 & -0.87 & -0.48 \\
20 & -0.86 & -0.45 \\
21 & -0.85 & -0.42 \\
22 & -0.84 & -0.39 \\
23 & -0.83 & -0.36 \\
24 & -0.80 & -0.33 \\
\hline & &
\end{tabular}

and 21 ), DTs between SSN and $I(\mathrm{GCR})$ and between TA and $I(\mathrm{GCR})$ are $\approx 13$ and $\approx 15$ months, respectively.

During even cycles, the correlations between SSN and $I(\mathrm{GCR})$ and TA and $I(\mathrm{GCR})$ are essentially similar. Chowdhury, Kudela, and Dwivedi (2013) noted that the DT between $I(\mathrm{GCR})$ and some solar indices, such as SSN or area and SFI, in Cycle 23 is remarkably large, reaching $\approx 18$ months. Previous investigations (e.g. Nagashima and Morishita, 1980a; Mavromichalaki, Belehaki, and Rafios, 1998; Mavromichalaki, Paouris, and Karalidi, 2007) of Cycles 17 to 22 showed an average DT of $\approx 2.4$ months in even cycles and of $\approx 12.4$ months for odd ones. According to the interpretation of 22-year patterns in the correlations between SSN and $I(\mathrm{GCR})$ and TA and $I(\mathrm{GCR})$ found by Cliver and Ling (2001) incoming GCRs during the $A>0$ epoch will be less affected by the drift effects due to increasing of TA at the beginning of an odd cycle or due to the diffusion associated with enhanced coronal mass ejection (CME) activity. At the beginning of even SA cycles for $A<0$, when GCRs approach the Sun along the HCS, they will be easily affected by the changes in the TA and low-latitude CMEs. Also, the interpretation by Van Allen (2000) is consistent with the drift effect, although the author rightly emphasizes the importance of the irregular fields at high latitudes (e.g. Balogh et al., 1999) and the small latitudinal gradients (Simpson et al., 1995) measured by Ulysses. 


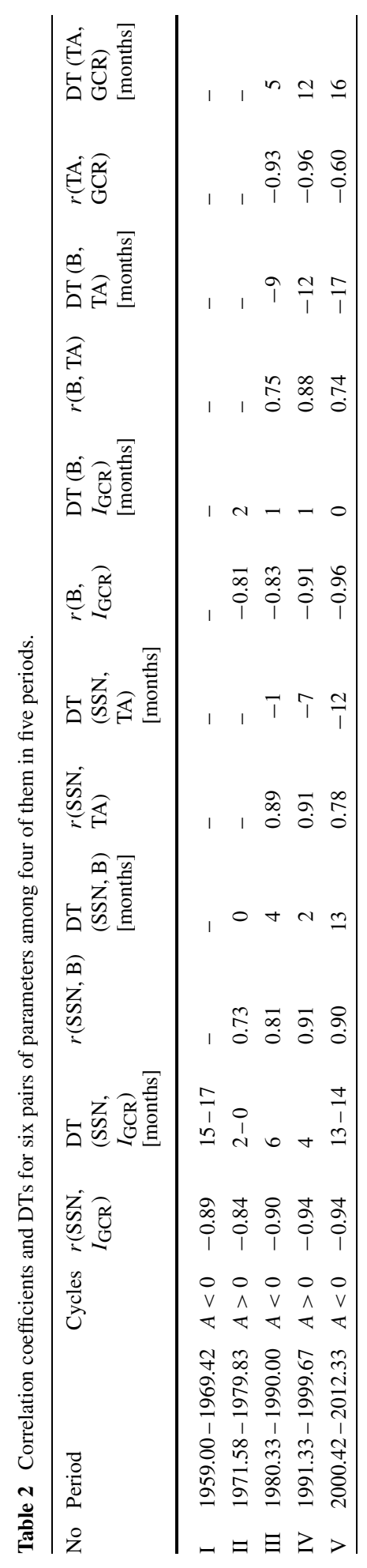




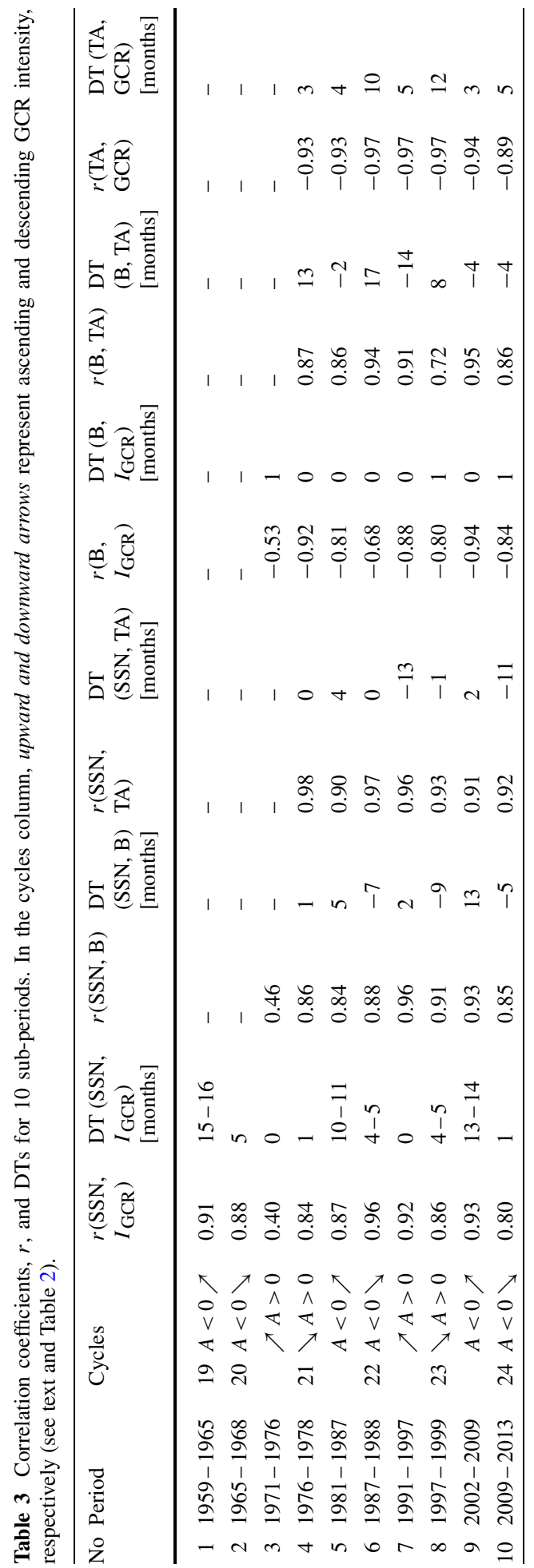




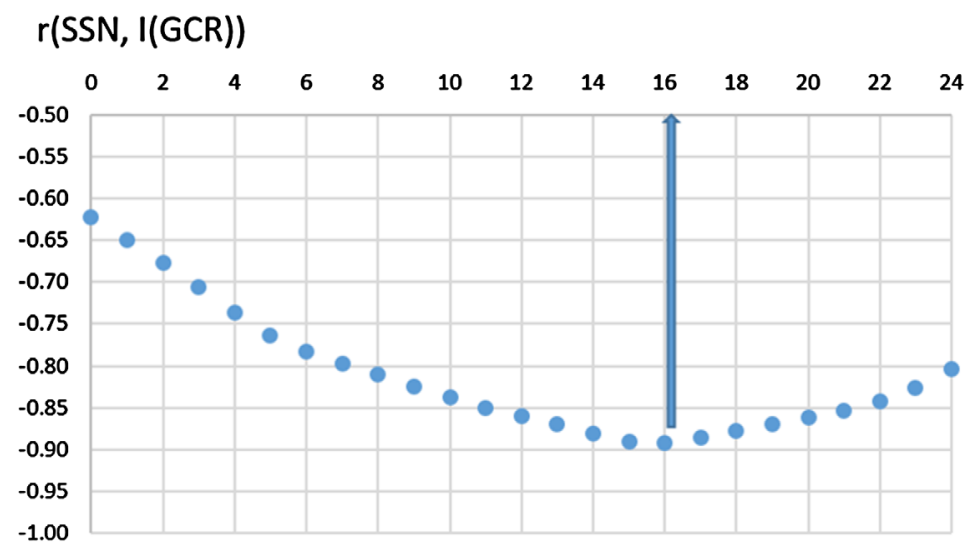

(a)
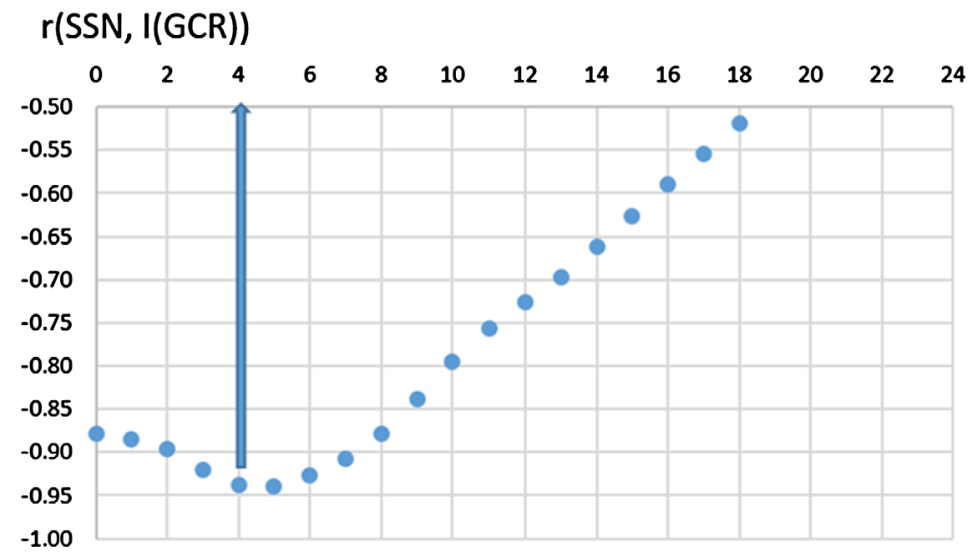

(b)

Figure 7 Correlation coefficients, $r$, for different monthly shifts $(0-24)$. Up-arrows show DTs obtained from the maximum absolute value of $r$ for periods (see text): (a) $1959-1969(A<0) \mathrm{DT}=(16 \pm 1)$ months. (b) $1991-1999(A>0) \mathrm{DT}=(4 \pm 1)$ months.

Chowdhury, Kudela, and Dwivedi (2013) and Chowdhury and Kudela (2018) discussed in detail the phenomena of GCR modulation and the DT problem between $I(\mathrm{GCR})$ and various parameters characterizing conditions in the heliosphere. Chowdhury, Kudela, and Dwivedi (2013) mentioned that this is a very complex phenomenon occurring throughout the heliosphere that depends on several factors. No single solar parameter can account for the GCR intensity variations. The IMF magnitude, $B$, plays a vital role in GCR modulation, because the Larmor radius (an important parameter determining particle transport in space) is inversely proportional to the strength of the IMF. An increase in the IMF strength decreases the Larmor radius and the diffusion coefficient, leading to an increase in GCR modulation. It is remarkable, however, that the highest correlation between $B$ and $I(\mathrm{GCR})$ occurs at zero DT in Cycle 23 (see DT ( $B, I(\mathrm{GCR})$ ) in Table 2). Chowdhury, Kudela, and Dwivedi (2013) proposed that there is no significant DT between $B$ and $I(\mathrm{GCR})$, because the local disturbances such as CMEs, traveling shocks, etc., which are injected into the inner heliosphere, dominate over the effects of merged interaction regions (MIRs) and globally 
merged interaction regions (GMIRs) operating at large distances in the outer heliosphere in this interval.

It is possible that some indices, such as SSN and SFI, represent global effects, whereas others, such as $B$ and Ap, represent local effects, limited to near the Earth on the ecliptic plane. This is consistent with Usoskin's et al. (1998) suggestion that the GCR modulation appears to be clearly correlated with only the global indices because of the complicated transport of GCRs in the heliosphere.

Cane et al. (1999) reported that the physical mechanisms responsible for the 22-year modulation of GCRs are time-dependent heliospheric drifts and outward propagating diffusive barriers. The relative importance of these two, fundamentally different, mechanisms changes with the SMC. Diffusive barriers are supposedly formed by CMEs, shocks, and high-speed flows at 10-15 AU, the above-mentioned MIRs. Drifts are expected to be most influential in the years when SA is lowest and the large-scale heliospheric magnetic field changes slowly. In such a case, diffusive barriers or GMIRs tend to be absent Cane et al. (1999).

In the present article, we examine the whole period from 1959 to 2014 which includes two and a half 22-year solar magnetic cycles, and we divided it into five periods according to their polarity (see Table 2). To examine the DTs in a more detailed way, each of these five periods were further divided into two sub-periods: ascending and descending periods of $I(\mathrm{GCR})$ (see Table 3). We observed the 22-year periodicity of the DT between SSN and $I(\mathrm{GCR})$ and SSN and $B$ but not between the remaining parameters (see Table 2). In particular, we observed the 22-year periodicity of the DT only between SSN and I(GCR), but we did not observe a significant DT between $B$ and $I(\mathrm{GCR})$, despite the high correlation between them (see Table 3). We think that the main reason for this phenomenon is essentially the temporal rearrangement of the structure of the IMF turbulence from the minimum to the maximum epoch of SA, which causes the structure of the IMF turbulence to show polarity dependence (Siluszyk et al., 2015).

\section{Conclusions}

1. Solar activity characterized by the global sunspot number (SSN) and its change determines the conditions prevailing in the heliosphere, i.e. variable IMF and its turbulence, HCS, CME, MIR, shocks. These changing conditions affect the GCR propagation in the heliosphere.

2. The structure of the IMF turbulence significantly changes from the minimum to the maximum epoch of SA and shows polarity dependence.

3. A 22-year variation of the DT between SSN and $I(\mathrm{GCR})$ was observed. DTs for periods $A<0$ are greater than DTs for periods $A>0$.

4. We also found that DTs in the $A<0$ epoch are larger in the ascending $I(\mathrm{GCR})$ period than in the descending $I(\mathrm{GCR})$ period. In the $A>0$ epoch, we did not find DTs in the ascending $I(\mathrm{GCR})$ period, but small DTs in the descending $I(\mathrm{GCR})$ period.

5. The main reasons for this phenomenon are essential temporal rearrangements of the structure of the IMF turbulence. The drift of GCRs caused by the gradient and curvature of the IMF is an additional factor, which strengthens this phenomenon.

6. To establish the properties of DTs from various cycles of SA, it is very important to model and experimentally analyze the propagation of cosmic ray particles in the heliosphere. 
Acknowledgements We are grateful to the data bases providing solar, interplanetary, geomagnetic, and NM data used in this paper. We used data from the websites: http://omniweb.gsfc.nasa.gov, http://spidr. ionosonde.net/spidr/, and http://www.nmdb.eu.

Disclosure of Potential Conflicts of Interest The authors declare that there are no conflicts of interest.

Publisher's Note Springer Nature remains neutral with regard to jurisdictional claims in published maps and institutional affiliations.

Open Access This article is distributed under the terms of the Creative Commons Attribution 4.0 International License (http://creativecommons.org/licenses/by/4.0/), which permits unrestricted use, distribution, and reproduction in any medium, provided you give appropriate credit to the original author(s) and the source, provide a link to the Creative Commons license, and indicate if changes were made.

\section{References}

Alania, M.V., Iskra, K., Siluszyk, M.: 2003, Experimental and theoretical investigations of the 11-year variation of galactic cosmic rays. Adv. Space Res. 32(4), 651. DOI. ADS.

Alania, M.V., Iskra, K., Siluszyk, M.: 2008a, On the new index of the long-period modulation of the galactic cosmic rays intensity. Acta Phys. Pol. B 39(11), 2961. ADS. http://www.actaphys.uj. edu.pl/fulltext?series $=$ Reg\&vol=39\&page $=2961$.

Alania, M.V., Iskra, K., Siluszyk, M.: 2008b, New index of long-term variations of galactic cosmic ray intensity. Adv. Space Res. 41(2), 267. DOI.

Alania, M.V., Iskra, K., Siluszyk, M.: 2010, On relation of the long period galactic cosmic rays intensity variations with the interplanetary magnetic field turbulence. Adv. Space Res. 45(10), 1203. DOI.

Alania, M.V., Aslamazashvili, R.G., Bochorishvili, T.B., Iskra, K., Siluszyk, M.: 2001, The role of drift on the diurnal anisotropy and on temporal changes in the energy spectra of the 11-year variation for galactic cosmic rays. Adv. Space Res. 27(3), 613. DOI.

Balogh, A., Forsyth, R.J., Lucek, E.A., Horbury, T.S., Smith, E.J.: 1999, Heliospheric magnetic field polarity inversions at high heliographic latitudes. Geophys. Res. Lett. 26, 631. DOI. ADS.

Burlaga, L.F., McDonald, F.B., Ness, N.F.: 1993, Cosmic ray modulation and the distant heliospheric magnetic field: Voyager 1 and 2 observation from 1986 through 1989. J. Geophys. Res. 98, 1. DOI.

Cane, H.V., Wibberenz, G., Richardson, I.G., von Rosenvinge, T.T.: 1999, Cosmic ray modulation and the solar magnetic field. Geophys. Res. Lett. 26(5), 565. DOI. ADS.

Chowdhury, P., Kudela, K.: 2018, Quasi-periodicities in cosmic rays and time lag with the solar activity at a middle latitude neutron monitor: 1982 - 2017. Astrophys. Space Sci. 363, 250. DOI.

Chowdhury, P., Kudela, K., Dwivedi, B.N.: 2013, Heliospheric modulation of galactic cosmic rays during Solar Cycle 23. Solar Phys. 286, 577. DOI.

Cliver, E.W., Ling, A.G.: 2001, 22 year patterns in the relationship of sunspot number and tilt angle to cosmicray intensity. Astrophys. J. Lett. 551(2), 189. DOI.

Dorman, L.I.: 1959, Proc. 6th ICRC 4, 328.

Dorman, L.I.: 1974, Cosmic Rays. Variations and Space Explorations, Elsevier, Amsterdam, New York.

Ferreira, S.F., Potgieter, M.S.: 2004, Long-term cosmic-ray modulation in the heliosphere. Astrophys. J. 603(2), 744. DOI.

Inceoglu, F., Knudsen, M.F., Karoff, C., Olsen, J.: 2014, Modeling the relationship between neutron counting rates and sunspot numbers using the hysteresis effect. Solar Phys. 289(4), 1387. DOI.

Iskra, K., Siluszyk, M., Alania, M.V.: 2015, Rigidity spectrum of the long-period variations of the galactic cosmic ray intensity in different epochs of solar activity. J. Phys. Conf. Ser. 632, 1. DOI.

Jokipii, J.R.: 1971, Propagation of cosmic rays in the solar wind. Rev. Geophys. Space Phys. 9, 27. DOI. ADS.

Jokipii, J.R., Kota, J.: 1996, 3D heliospheric simulations of cosmic rays in the light of Ulysses. Nuovo Cimento C 19(6), 921. DOI.

Jokipii, J.R., Thomas, B.: 1981, Effects of drift on the transport of cosmic rays. IV - Modulation by a wavy interplanetary current sheet. Astrophys. J. 243, 1115. ADS.

Le Roux, J.A., Potgieter, M.S.: 1995, The simulation of complete 11 and 12 year modulation cycles for cosmic rays in the heliosphere using a drift model with global merged interaction regions. Astrophys. $J$. 442, 847. ADS.

Lockwood, J.A., Webber, W.R.: 1984, Observations of the dynamics of the cosmic ray modulation. J. Geophys. Res. 89, 17. DOI. 
Mavromichalaki, H., Belehaki, A., Rafios, X.: 1998, Simulated effects at neutron monitor energies: evidence for a 22-year cosmic-ray variation. Astron. Astrophys. 330, 764. ADS.

Mavromichalaki, H., Paouris, E., Karalidi, T.: 2007, Cosmic-ray modulation: an empirical relation with solar and heliospheric parameters. Solar Phys. 245, 369. DOI.

Nagashima, K., Morishita, I.: 1980a, Long-term modulation of cosmic rays and inferable electromagnetic state in solar modulation region. Planet. Space Sci. 28(2), 177. DOI.

Nagashima, K., Morishita, I.: 1980b, Twenty-two year modulation of cosmic rays associated with polarity reversal of polar magnetic field of the Sun. Planet. Space Sci. 28(2), 195. DOI.

Parker, E.N.: 1963, Interplanetary Dynamical Processes. Interscience Monographs and Texts in Physics and Astronomy, 8. Interscience Publishers, New York. ADS.

Sierra-Porta, D.: 2018, Cross correlation and time-lag between cosmic ray intensity and solar activity during solar cycles 21, 22 and 23. Astrophys. Space Sci. 363(7), 137. DOI.

Siluszyk, M., Iskra, K., Alania, M.V.: 2014, Rigidity dependence of the long period variations of galactic cosmic ray intensity: a relation with the interplanetary magnetic field turbulence for 1968-2002. Solar Phys. 289(11), 4297. DOI. ADS.

Siluszyk, M., Iskra, K., Alania, M.V.: 2015, 2-D modelling of long period variations of galactic cosmic ray intensity. J. Phys. Conf. Ser. 632, 012080. DOI.

Siluszyk, M., Wawrzynczak, A., Alania, M.V.: 2011, A model of the long period galactic cosmic ray variations. J. Atmos. Solar-Terr. Phys. 73(13), 1923. DOI. ADS.

Siluszyk, M., Iskra, K., Modzelewska, R., Alania, M.V.: 2005, Features of the 11-year variation of galactic cosmic rays in different periods of solar magnetic cycles. Adv. Space Res. 35(4), 677. DOI. ADS.

Siluszyk, M., Iskra, K., Alania, M.V., Miernicki, S.: 2015, Features of the interplanetary magnetic field turbulences in different periods of solar magnetic cycles. In: Conf. ICRC, Proceedings of Science. https://pos.sissa.it/236/125/pdf.

Siluszyk, M., Iskra, K., Alania, M.V., Miernicki, S.: 2018, Interplanetary magnetic field turbulence and rigidity spectrum of the galactic cosmic rays intensity variation (1969-2011). J. Geophys. Res. 123(1), 30. DOI.

Simpson, J.A., et al:: 1995, Cosmic ray and solar particle investigations over the South polar regions of the Sun. Science 268(5213), 1019. DOI. ADS.

Singh, P.R., Saxena, A.K., Tiwari, C.M.: 2018, Mid-term periodicities and heliospheric modulation of coronal index and solar flare index during solar cycles $22-23$. J. Astrophys. Astron. 39, 1. DOI.

Usoskin, I.G., Kananen, H., Mursula, K., Tanskanen, P., Kovaltsov, G.A.: 1998, Correlative study of solar activity and cosmic ray intensity. J. Geophys. Res. 103(A5), 9567. DOI. ADS.

Van Allen, J.A.: 2000, On the modulation of galactic cosmic ray intensity during solar activity cycle 19, 20, 21, 22 and early 23. Geophys. Res. Lett. 27(16), 2453. DOI. 